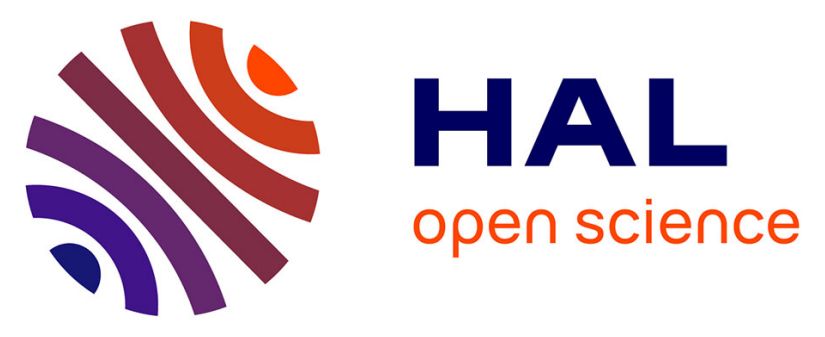

\title{
An Implantable, Low-Power Instrumentation for the Long Term Monitoring of the Sleep of Animals under Natural Conditions
}

Bertrand Massot, Niels C Rattenborg, Anders Hedenström, Susanne Akesson, Paul-Antoine Libourel

\section{To cite this version:}

Bertrand Massot, Niels C Rattenborg, Anders Hedenström, Susanne Akesson, Paul-Antoine Libourel. An Implantable, Low-Power Instrumentation for the Long Term Monitoring of the Sleep of Animals under Natural Conditions. 2019 41st Annual International Conference of the IEEE Engineering in Medicine \& Biology Society (EMBC), Jul 2019, Berlin, Germany. pp.4368-4371, 10.1109/EMBC.2019.8856359 . hal-02352897

\section{HAL Id: hal-02352897 https://hal.science/hal-02352897}

Submitted on 7 Nov 2019

HAL is a multi-disciplinary open access archive for the deposit and dissemination of scientific research documents, whether they are published or not. The documents may come from teaching and research institutions in France or abroad, or from public or private research centers.
L'archive ouverte pluridisciplinaire HAL, est destinée au dépôt et à la diffusion de documents scientifiques de niveau recherche, publiés ou non, émanant des établissements d'enseignement et de recherche français ou étrangers, des laboratoires publics ou privés. 


\title{
An Implantable, Low-Power Instrumentation for the Long Term Monitoring of the Sleep of Animals under Natural Conditions
}

\author{
Bertrand Massot ${ }^{1}$, Member, IEEE, Niels C. Rattenborg ${ }^{2}$, Anders Hedenstrom ${ }^{3}$, \\ Susanne Åkesson ${ }^{3}$ and Paul-Antoine Libourel ${ }^{4}$,
}

\begin{abstract}
Sleep is a universal and complex state and it is widely agreed that this state is present in every animal species. However, the evolutionary origins of sleep remain ignored or misunderstood, which has led researchers to study, in various species, this common behaviour of all living organisms. Sleep is commonly studied at various levels under laboratory conditions, using tethered devices which record electroencephalographic or electromyographic readings. These artificial settings tend to induce stress, reduce animal freedom and prevent the use of sleeping shelters. In this paper, we present a novel, implantable instrumentation for a complete characterization of sleep under natural conditions suitable for a wide range of animal species, even for animals as small as pigeons or mice. Several configurations of this system are possible to enable the measurement of up to 16 electrophysiology channels, 3 temperature channels as well as 3-axes accelerometry. With an embedded flash memory card for the storage of data collected, the system can be used as a datalogger for the recording of signals in the field.
\end{abstract}

\section{INTRODUCTION}

Classically, sleep is studied on laboratory animal models such as rats, mice, zebrafish, etc. Optogenetics, immunohistochemistry, behavioral tests, neuronal activity recordings, temperature measurements, electrophysiological studies (EEG, EMG, ECG, EOG) are various methods used to assess different aspects of sleep. However, most of these techniques involve restraining the animal which can induce stress and/or modify the natural sleep behaviour which would occur in a natural environment [1]. These restraints include, for example:

- The use of tethered devices;

- A reduction in the animal's freedom of movement;

- The preclusion of using sleeping shelters.

Portable, non-obtrusive solutions are therefore sought to enable the recording of signals under more natural conditions in order to reduce stress and preserve natural sleep patterns. Unfortunately, only a very few systems exist commercially for the recording of electrophysiological signals outside the

\footnotetext{
${ }^{1}$ Bertrand Massot is with Lyon Institute of Nanotechnology, UMR CNRS 5270, INSA Lyon, University of Lyon, 69100 Villeurbanne, France. bertrand.massoteinsa-lyon. fr

${ }^{2}$ Niels C. Rattenborg is with the Max Planck Institute for Ornithology, Avian Sleep Group, 82319 Seewiesen, Germany. rattenborg@orn.mpg.de

${ }^{3}$ Susanne Åkesson and Anders Hedenstrom are with the Department of Biology, Center for Animal Movement research, Lund University, SE-223 62 Lund, Sweden. susanne.akessonabiol.lu.se, anders.hedenstromebiol.lu.se

${ }^{4}$ Paul-Antoine Libourel is with the Lyon Neuroscience Research Center, UMR CNRS 5292, UMR INSERM U1028, UCBL, University of Lyon, 69008 Lyon, France. libourelesommeil.univ-lyonl. fr
}

laboratory. A method currently adopted by researchers in this area consists in the development and use of miniature loggers comprising an analog frontend for signal amplification and conditioning, analog-to-digital converters and an embedded storage media; the whole system being powered by a battery. The challenge is to produce an ultra-low power instrument which is as small as possible to be attached to the electrodes implanted in the animal. While several radiofrequency devices have been developed over the past 20 years (for example [2]-[5]), only one logger appears to be well suited for the recording of EEG and EMG activity under natural conditions [6]. However, this system may not achieve the autonomy necessary to record multiple sleep/wake cycles, due to battery lifetimes of less than 2 days. In a previous work [7], we presented a miniature, portable system for the recording of signals dedicated to the study of sleep patterns with the advantages of:

- Being versatile (both capable of RF communication and data logging);

- Being configurable (up to 26 channels for electrophysiology with independent sampling frequency);

- Integrating a complete range of tools for behavioral, electrophysiology and metabolic measurements with the addition of an embedded, sleep deprivation system;

- Battery lifetimes longer than 48 hours.

While having the advantages of a large versatility, in terms of adaption to multiple protocols and experimental settings, this device was still oversized in order to make it implantable.

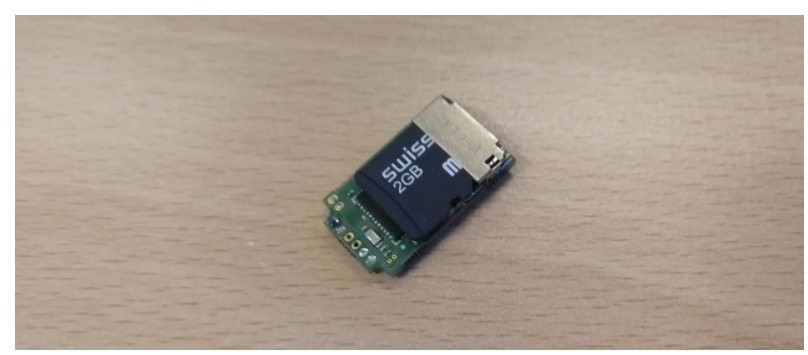

Fig. 1. Picture of the new miniature logger device

In this paper we present an adaptation of this former system which benefit froms multimodal measurements within a miniaturised electronic device for the logging of data under natural conditions. The system's single, $20 \mathrm{~mm} \times 10$ $\mathrm{mm}$ electronic board (Fig. 1) hosts a dedicated, 16 channel amplifier and analog-to-digital converter for electrophysiological signals. This frontend is coupled with an ultra-low 
power microcontroller unit which sequences, collects and transfers data from the electrophysiological frontend, the temperature measurement circuits and the 3-axis accelerometer. These data are stored on an embedded micro-SD flash card specifically chosen for its low energy consumption and its reliability when operated under extreme environments. Moreover, the system integrates a real-time clock, which maintains a calendar up to date in order to program the initial system startup and the periodic intervals of operation, thus extending the battery life even further.

\section{SYSTEM DESIGN}

\section{A. Electronic architecture of the system}

The electronic board of the system includes a RHD2116 integrated analog frontend (Intan Technologies LLC, Los Angeles, California). This frontend comprises 16 differential amplifiers with programmable bandpass filters and a 16-bit, 1 Msps, analog-to-digital converter. These 16 channels are dedicated to electrophysiological measurements (EEG, ECG, EOG, EMG) and the electrodes are connected directly on the input of each channel. The gain of each channel is fixed at $192 \mathrm{~V} / \mathrm{V}$, hence the resolution is $0.195 \mu \mathrm{V}$ over the range $\pm 6.4 \mathrm{mV}$. The frontend also contains three additional, nonamplified channels that are used for the sampling of temperature measurements. The conditioning circuits for temperature were conveniently based on polarisation resistors normally used for NTC thermistors in order to reduce the size and consumption of the circuit (Fig. 2). The ambient temperature is measured by a NTCG104ED104DT1X $100 \mathrm{k} \Omega$ chip NTC thermistor (TDK Corporation, Tokyo, Japan), which is placed directly on the electronic board. This thermistor is polarised with a $510 \mathrm{k} \Omega$ parallel resistor $R_{2}$ and a $120 \mathrm{k} \Omega$ series resistor $R_{1}$ to provide a linear output with an error less than $0.2{ }^{\circ} \mathrm{C}$ over the range $5-35^{\circ} \mathrm{C}$ and less than $1{ }^{\circ} \mathrm{C}$ over the range $0-40{ }^{\circ} \mathrm{C}$.
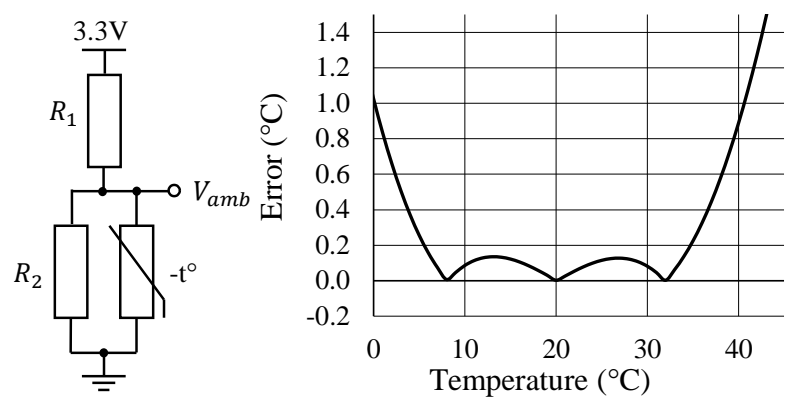

Fig. 2. Conditioning circuit for ambient temperature thermistor and theoretical absolute error associated with the linear model.

Implantable micro-NTC thermistors, e.g. the 223F312207U015 (Semitec USA Corporation, Torrance, California), can be used for their very small size $(0.3 \mathrm{~mm}$ diameter $)$ and encapsulation for in-body temprature measurements. When polarised with a single $10 \mathrm{k} \Omega$ series resistor $R$, the voltage obtained is linear with temperature over the range $25-45^{\circ} \mathrm{C}$ with an error less than $0.05{ }^{\circ} \mathrm{C}$ (Fig. 3).
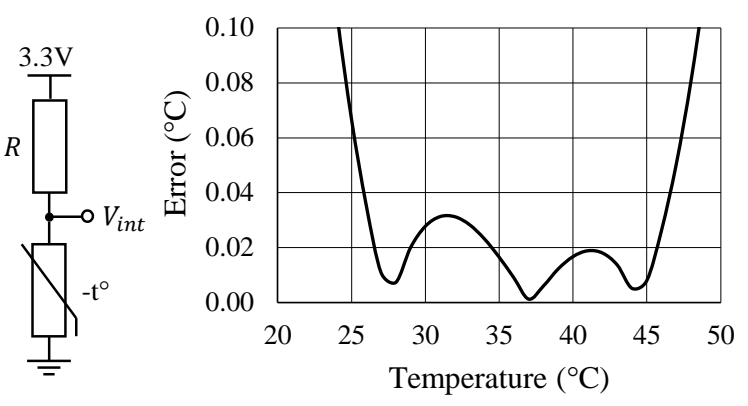

Fig. 3. Conditioning circuit for implanted temperature thermistors and theorical absolute error associated with the linear model.

A digital accelerometer ADXL362 (Analog Device Incorporation, Norwood, Massachusetts) is used for the measurement of acceleration along 3 orthogonal axes. This accelerometer has the advantage of a very low energy consumption during measurement which is less than $10 \mu \mathrm{W}$ with an output data rate of $400 \mathrm{~Hz}$. The range of measurement can be selected between $\pm 2 \mathrm{~g}, \pm 4 \mathrm{~g}$ or $\pm 8 \mathrm{~g}$, with a fixed resolution of 12 bits, leading to a resolution as small as 0.001 $\mathrm{g}, 0.002 \mathrm{~g}$ and $0.004 \mathrm{~g}$, respectively.

Both the integrated analog frontend as well as the digital accelerometer are connected to a microcontroller unit (MCU) through serial peripheral interfaces (SPI) running at $8 \mathrm{Mbps}$. The MCU used in this system is a MSP430FR5964 (Texas Instruments, Dallas, Texas) which has been chosen because it contains $256 \mathrm{kB}$ of internal ferroelectric random access memory (FRAM) and a real-time clock (RTC). The system benefits from this high memory size (considering this class of MCU) as it enables the allocation of space for a large buffering of data before writing on the flash memory. Indeed, the access and internal management of SD cards generates additional energy consumption which can be reduced by writing multiple sectors consecutively. The RTC is used to run a calendar to program dates and periods of measurements, as explained in the next section. Finally, this MCU has the advantage of low energy consumption $(100 \mu \mathrm{A} / \mathrm{MHz}$ in active mode and less than $1 \mu \mathrm{A}$ with active RTC when operating under 3.3 Volts).

Regarding power consumption, microSD cards can largely vary and particular care must be taken when choosing a specific model for use with the system. Examples of suitable models are industrial microSD cards such as the S-300u series from Swissbit (Swissbit, Bronschhofen, Swiss) for 2 GB models, or the U300 series from Delkin Devices (Delkin Devices, San Diego, California) for 8 GB models. These models have been chosen as they have guaranteed maximum current consumption for read, write and idle operations as reported in Table I.

For additional energy saving when the system is shut down while the RTC of the MCU remains active for future wakeup, a P-channel MOSFET transistor is used as a power switch for peripherals (RHD2116, ADXL362 and SD card). This enables the complete removal of standby currents, which can be particularly high, depending on the SD card model used 
TABLE I

CURRENT CONSUMPTION OF SELECTED MODELS FOR MICROSD FLASH MEMORY CARDS.

\begin{tabular}{|c|c|c|c|}
\hline SD card series & Idle & Read & Write \\
\hline S-300u, $2 \mathrm{~GB}$ & $0.15 \mathrm{~mA}$ & $50 \mathrm{~mA}$ & $60 \mathrm{~mA}$ \\
$\mathrm{U} 300,8 \mathrm{~GB}$ & $0.5 \mathrm{~mA}$ & $50 \mathrm{~mA}$ & $100 \mathrm{~mA}$ \\
\hline
\end{tabular}

as shown in Table I. The current consumption of the system is given in Table II for the different operating modes. While the current consumption remains identical in shutdown mode (as all peripherals are powered off), it can largely vary when awake, depending on the overall sampling rate and SD card type. The consumption presented when awake is the mean consumption of the system comprising data sampling and transfer to SD card.

TABLE II

CURRENT CONSUMPTION OF THE SYSTEM UNDER 3.3 VOLTS.

\begin{tabular}{|c|c|c|c|}
\hline SD card series & Sampling rate & Shut down & Awake (average) \\
\hline S-300u $(2 \mathrm{~GB})$ & $13 \times 128 \mathrm{sps} / \mathrm{s}$ & & $1.12 \mathrm{~mA}$ \\
U300 $(8 \mathrm{~GB})$ & $13 \times 128 \mathrm{sps} / \mathrm{s}$ & $1.7 \mu \mathrm{A}$ & $1.93 \mathrm{~mA}$ \\
S-300u $(2 \mathrm{~GB})$ & $13 \times 1024 \mathrm{sps} / \mathrm{s}$ & & $2.53 \mathrm{~mA}$ \\
U300 $(8 \mathrm{~GB})$ & $13 \times 1024 \mathrm{sps} / \mathrm{s}$ & & $5.42 \mathrm{~mA}$ \\
\hline
\end{tabular}

Figure 4 shows a functional diagram of the entire hardware involved in the system.

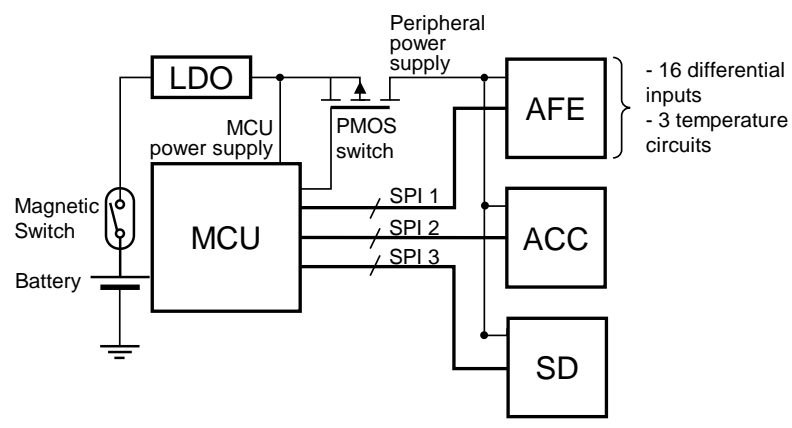

Fig. 4. Functional diagram of the system. A P-channel MOSFET is used as a controlled power switch for peripherals to ensure minimal power consumption during shut down periods.

\section{B. Embedded software}

The software embedded in the MCU (firmware) executes the following tasks :

1) Configures the analog frontend and the digital accelerometer;

2) Sets cyclic awake and shut down periods using alarms from the calendar;

3) Collects data from electrophysiological, temperature and accelerometric channels and stores them temporarily in the internal FRAM;

4) Transfers buffers of data from FRAM to SD card.

The number of channels for electrophysiological, temperature and accelerometric data, as well as the sampling rate (common for every channel) are defined in the firmware at programming time. A measurement cycle is defined by the duration of a period of measurement (from one minute up to indefinite) and the duration of a shutdown period. Initially, the system enters a low-power mode at boot and monitors periodically for the insertion of a micro SD card in the dedicated slot (Fig. 5).

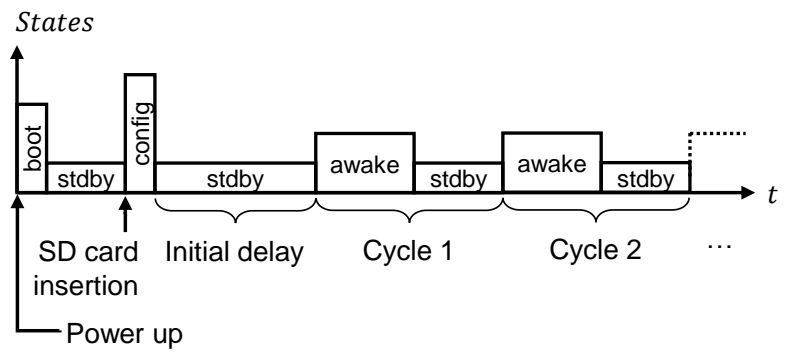

Fig. 5. Chronogram of successive states of the device. When SD card is inserted, a programmable delay enables postponing the beginning of the measurement cycles. All cycles consist of an "awake" state where signals are recorded, and a "standby" state where the system is shut down to save power. Duration of periods is programmable.

Other parameters such as the current date, the initial date of measurement following the insertion of the SD card, the period of active measurement, the duration of shutdown and the number of cycles are written into the SD card before its insertion into the system. These parameters provide a advantageous flexibility when recording signals from animals under natural conditions and extends the autonomy of the system. Indeed, when recording specific periods of the year, for example for migratory birds, the system can remain in a very low power state to save battery energy by setting a programmed alarm in the real-time calendar to only start the measurements at a specific date. Also, when very long experiments are desired (over a year for example), cyclic measurements (a few hours per day or a few days per month) can also be programmed in order to reduce the system consumption by using cycles of measurement. This enables the use of the system with a smaller battery in order to reduce the overall size, and more importantly, its weight (especially for small animals such as birds).

\section{EXPERIMENTAL RESULTS}

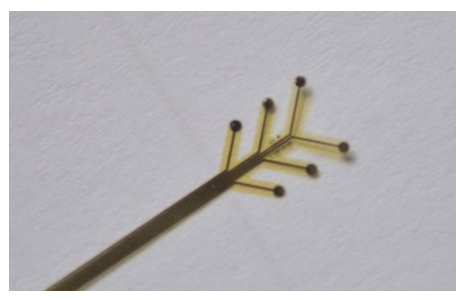

(a)

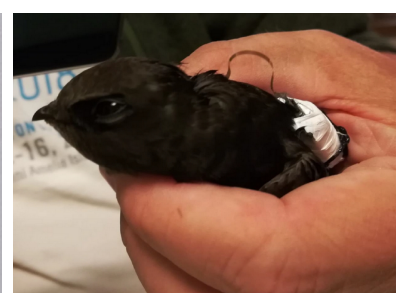

(b)
Fig. 6. (a) Flexible printed electrodes array for subcutaneous EEG and (b) picture of a common swift with the system attached to its back.

Following in-laboratory characterization and the verification of proper operation of the system, several experiments 

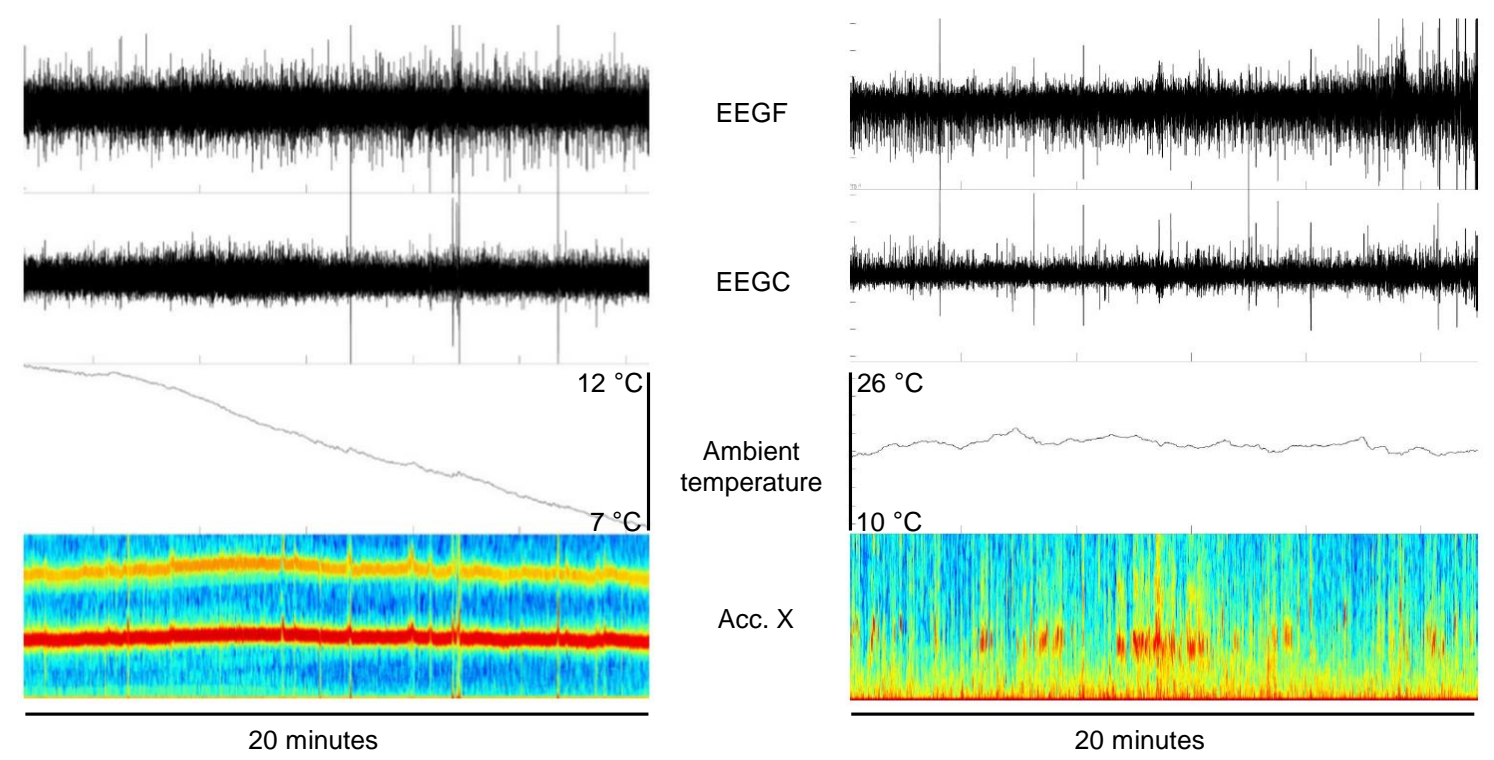

Fig. 7. Exemples of signals obtained during a continuous, 48 hour period of recording on a common swift under natural conditions during flight (left) and at rest (right).

were carried out in the field to confirm the ability of the system to acquire signals on animals under natural conditions.

One of these experiments was carried out on adult common swifts (Apus apus) captured at breeding sites in southeastern Sweden, which were recorded over 48 hours while left free-flying in their natural habitat. After the implantation of subcutaneous, flexible, printed electrodes (Fig. 6a), the system was connected to the flexible electrode circuit using a zero-insertion force (ZIF) connector, and finally attached on the back of the animal using a full body harness (Fig. 6b) [8].

Permission to capture common swifts was given by the landowners involved, the Swedish Environmental Protection Agency and the Swedish Ringing Centre at the Swedish Museum of Natural History (no. 440) to Susanne Åkesson. Ethical permission for field work on logged common swifts in Sweden was given by Malmö-Lunds djurförsöksetiska nämnd (Dnr 5.8.18-06330/2018) to Susanne Åkesson.

Figure 7 illustrates signals that were obtained from common swifts during the experiment. On the left, it can be seen on the accelerometer spectrogram that the bird is flying with continuous wing-beats. Additionally, the decrease in temperature over the 20 minute recording clearly indicates a rising elevation, i.e the bird was climbing. On the right, one can observe alternate flap-gliding flight. The EEG signal thus indicates an animal in an awaked state during this period. This example demonstrates the ability of the system to provide physiological information and combining behavioral and environmental measurements on a freely moving animal under natural conditions.

\section{CONCLUSion}

In conclusion, this miniature device dedicated to record physiological, behavioral and environmental signals furnishes opportunities for further experiments which were not possible to undertake before. Future works will concentrate on the reliability of the system especially, when used as an implant regarding the sealing and biocompatibility of the casing. In research concerning sleep studies, the device will provide signals relevant to the analysis of sleep/wake cycles with the advantage of being implantable, thus enabling monitoring of the animal in its natural environment, and, as a consequence, reducing induced stress and favoring more natural sleep patterns.

\section{REFERENCES}

[1] X. Tang, S. M. Orchard, X. Liu, and L. D. Sanford, "Effect of varying recording cable weight and flexibility on activity and sleep in mice," Sleep, vol. 27, no. 4, pp. 803-810, 2004.

[2] D. Lapray, J. Bergeler, E. Dupont, O. Thews, and H. J. Luhmann, "A novel miniature telemetric system for recording eeg activity in freely moving rats," Journal of Neuroscience Methods, vol. 168, no. 1, pp. $119-126,2008$.

[3] X. Tang and L. D. Sanford, "Telemetric recording of sleep and home cage activity in mice," Sleep, vol. 25, no. 6, pp. 677-685, 2002.

[4] M. Weiergräber, M. Henry, J. Hescheler, N. Smyth, and T. Schneider, "Electrocorticographic and deep intracerebral eeg recording in mice using a telemetry system," Brain research protocols, vol. 14, no. 3, pp. 154-164, 2005.

[5] A. Zayachkivsky, M. J. Lehmkuhle, J. H. Fisher, J. J. Ekstrand, and F. E. Dudek, "Recording eeg in immature rats with a novel miniature telemetry system," Journal of neurophysiology, vol. 109, no. 3, pp. 900911, 2012.

[6] A. L. Vyssotski, A. N. Serkov, P. M. Itskov, G. Dell'Omo, A. V. Latanov, D. P. Wolfer, and H.-P. Lipp, "Miniature neurologgers for flying pigeons: multichannel eeg and action and field potentials in combination with gps recording," Journal of neurophysiology, vol. 95, no. 2, pp. 1263-1273, 2006.

[7] B. Massot, S. Arthaud, B. Barrillot, J. Roux, G. Ungurean, P.-H. Luppi, N. C. Rattenborg, and P.-A. Libourel, "Oneiros, a new miniature standalone device for recording sleep electrophysiology, physiology, temperatures and behavior in the lab and field," Journal of neuroscience methods, 2018

[8] S. Åkesson, R. Klaassen, J. Holmgren, J. W. Fox, and A. Hedenström, "Migration routes and strategies in a highly aerial migrant, the common swift apus apus, revealed by light-level geolocators," PLoS One, vol. 7, no. 7, p. e41195, 2012. 